\title{
THE USE OF ACYCLOVIR AND PHYLLANTHUSNIRURI SUPPLEMENT AS MEDICATION FOR RECURRENT INTRAORAL HERPES INFECTIONS (SERIAL CASE REPORTS)
}

\author{
Marcia $^{1 *}$, SitiAliyah Pradono ${ }^{2}$
}

1. Postgraduate Program in Oral Medicine, Faculty of Dentistry, Universitas Indonesia, Jakarta, Indonesia

2. Department of Oral Medicine, Faculty of Dentistry, Universitas Indonesia, Jakarta, Indonesia

*Email: marcia_vip@yahoo.com

\begin{abstract}
Recurrent intraoral herpes infection (RIH) is one of the recurrent herpes simplex virus type-1 infections (HSV-1). It presents as single or clustered painful ulcers occur mainly on the keratinized mucosa, with the size of one to five millimeter. The Onset of RIH is considered to be associated with various endogenous and exogenous triggers. Therefore, it is important to recognize each individual triggers to prevent and to minimize and the infection for recurring. This report will describe three cases of RIH associated with emotional and physical stress. All patients have experienced continous recurrence of the disease for almost a year. Symptoms included painful multiple minute ulcers on oral mucosa initiated with fever. Serology investigation on antibody against HSV-1 confirmed a reactive elevation in these patients. Treatment consisted of acyclovir per oral $1000 \mathrm{mg}$ daily per oral, counseling on stress condition and intake of nutritional supplementation. Nevertheless, stress conditions were difficult to overcome, therefore long-term medications were recommended to prevent recurrence episodes. One patient was taken a long-term acyclovir therapy and the other two patients were taken long-term acyclovir therapy with Phyllanthusniruri (Meniran) supplementation. Follow-ups on three cases showed the benefit of taking Phyllanthusniruri (Meniran) supplementation as reduction in RIH symptoms, shorten healing time and prolonged recurrence interval.
\end{abstract}

Keywords: Recurrent intraoral herpes, preventive, stress, nutritional, acyclovir, Phyllanthusniru

\section{Introduction}

Cases of oral herpes virus infections are frequently found by medical and dental professionals. The infections might be caused by herpes simplex virus type $1(\mathrm{HSV}-1)$ and/or type 2 (HSV-2). ${ }^{1}$ In general, oral HSV infections were caused by HSV-1, 50 percent of primary infection are subclinical. ${ }^{2}$ Approximately 30 to 40 percent of patients who have been exposed to HSV will develop recurrent infections. ${ }^{3}$ Recurrent intraoral herpes (RIH) is intraoral recrudescence of herpes simplex (HSV-1) infections presents as one to five mm single or clustered painful ulcers with a bright erythematous border occurs mainly on the keratinized mucosa. ${ }^{4}$ Reactivation of latent HSV-1 might be related to breakdown in local immunosurveillance or an alteration in local inflammatory mediators. Thus, reactivated HSV-1 will migrates from the latent site to peripheral tissue. ${ }^{4,5}$ Many authors have been noted several endogenous triggers, such as immune suppression condition or vitamin deficiency, emotional and physical stress, menstruation illness and fatigue; and several exogenous triggers, such as ultraviolet radiation tissue damage, severe cold and hot weather and trauma to the mouth. ${ }^{3,6,7}$ Emotional stressas stated by the patients is the most frequent trigger to the onset. ${ }^{6}$ Recurrent infection is related with a rise in $\mathrm{IgG}$ antibody titer. ${ }^{4}$ Recurrent intraoral herpes is a self-limiting disease and treatment for RIH is primarily symptomatic such as adequate fluid intake, analgesic/antipyretics and local antiseptics., 4, 8 Some clinicians advocate the use of suppressive doses of antiviral therapy to reduce illness. ${ }^{1}$ When necessary, nutritional supplementation must be stressed to the patient. ${ }^{3}$ It is important for each patient to maintain their immune system also recognize their individual recurrence triggers to minimize or prevent recurring infection. ${ }^{6}$ Few strategies were stated such as, stress reduction, daily supplementation and long term antiviral therapy. ${ }^{6,9,10}$ 
This present serial case will describe three RIH induced by stress and the usage of long term acyclovir therapy and Phyllanthusniruri (P.niruri) extract supplement as one of strategies to prevent recurrence episodes.

\section{Serial Case Reports}

\section{Case 1}

A 29-year-old woman visited oral medicine clinic with chief complaint of recurrent ulcers for over a year. She had seeking for treatment eight months ago, the reactive anti HSV-1 IgG was 3.68 (reactive) and she was diagnosed with a HSV-1 infection. She received medication of antiviral (Acyclovir) painkiller (acetaminophen) and 10 percent povidone iodine mouthrinse. However, the symptom remained after the treatment as multiple small ulcers on her tongue and gingival. She also reported havinga fever and many sleepless nights. Extra oral examination revealed painless tender swelling of submandibular lymph node. Intraoral examination identified multiple regular and irregular ulcers on maxilla labial mucosa, left buccal mucosa, left lateral side of the tongue and lingual mandible attached gingival; approximately 4-6mm in diameters with a bright erythematous border. As the condition above, she was diagnosed by RIH infection. The new treatment included the oral administration of antiviral therapy (acyclovir $200 \mathrm{mg}$ five times a day), symptomatic therapies included theuse of Aloclair ${ }^{\circledR}$ mouthrinse and the oral intake of nutritional supplements. Education on pathogenesis of the disease, emphasis the importance of having enough rest and nutritional food were provided. On one week follow up, other minute ulcers developed on left buccal mucosa, mandible attached gingival and tongue the day before. The interval onset of RIH was 3 days after the first episode was healed. Serological examination showed the level of reactive antiHSV-1 IgG was 3.43. She was suggested to continue the medication. On day 16, she experienced the third onsetafter 5 days interval. She shared about her emotional stresses, fatigue and irregular medication intakes. The preventive strategies for her case were emotional and physical stress reduction, nutritional food and long term antiviral therapy (Acyclovir 200mg 5 times daily). Education was put forward to emphasis the patient compliance to take medicine regularly for long period of time and to have routine serological examination follow-ups. On her last visit (day 30), she was having the fourth onset after 10 days interval. There were symptoms reduction and rapid healing on the third and fourth onset. The antiviral therapy was continued with the additional multivitamin and zinc (Surbex Z®) supplementation. Two months after the last visit, she reported that sometimes ulcers still reoccurred with a decrease in ulcers quantity, faster healing and extended interval of onsets. (Figure 1.)

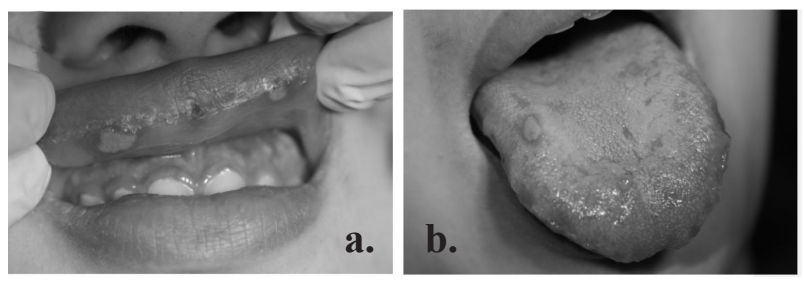

Figure 1. Ulcers developed on (a)labial mucosa and (b) tongue. (Case 1)

\section{Case 2}

A 39-year-old man visited the oral medicine clinic with the complaints of recurrent ulcers for a year and sometimes had fever 1-2 days before disease onset. He had seeking for treatment and receiving medication with steroid (methylprednisone), antifungal (nystatin oral suspension) and topical anesthesia solution. However the ulcers reoccurred after the medication was ended. He stated having emotional stress and sleeplessness for a year. Extra oral examination revealed painless tender swelling of submandibular lymph node. Intraoral examination identified multiple minute regular ulcers on labial mucosa and ventral of the tongue with bright erythematous border. Based on the history and clinical examination, he was suspected having a RIH The antiHSV-1 and antiHSV-2 titer were examined. Medications included antiviral therapy (acyclovir 200 mg five times a day) and an additional multivitamin and zinc (Surbex $Z ®$ ) supplement. He was informed about the pathogenesis of the disease, the importance of having adequate rest, taking nutritional food and reduction of stress. On one week follow up, the ulcers was healed, serologic examination showed a reactive antiHSV-1 IgG (3.92), it confirmed the diagnosis as a RIH. As a preventive strategy of the recurrence episodes, P.niruri extract supplement was suggested three times daily and management of stress. Unfortunately, on day 21, he had another recurrence with 14 days interval and again he was given antiviral therapy (acyclovir 200mg five times a day). P.niruriextractsupplement was continued. He believed emotional stress had cause the disease onset. Another recurrence occurs on day 45 within 17 days interval with lower reactive antiHSV-1 IgG (1.67) and healed after 5 days. On day 85 follow up, he never experienced any recurrence. He reported had taken P.niruri extract regularly and successfully reducing his stress. Two months after his last visit, his condition improved. He stated had got only one recurrent episode in 2 months period, with less amount of developing ulcer, shortens healing time. (Figure 2.) 

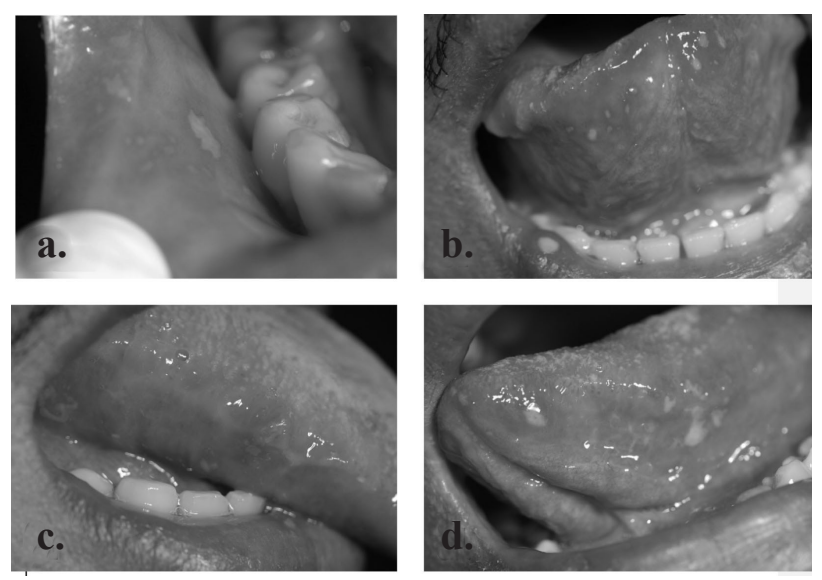

Figure 2. Multiple ulcers on (a)buccal mucosa, (b)ventral and (c-d)lateral of the tongue. (Case 2)

\section{Case 3}

A 44-year-old man visited the oral medicine clinic with complaints of recurrent multiple ulcers for almost 3 months and always come with fever 1-2 days before disease recurrence. He reported having physical and emotional stress for the past 3 months due to family financial problems. Extraoral examination revealed painless hard swelling of submandibular lymph node. Intraoral examination identified multiple regular minute ulcers spreading on oral mucosa and $6 \mathrm{~mm}$ in diameter irregular ulcers on labial mucosa. Each ulcer surrounded with a bright erythematous border. He was then suspected of having a RIH and receiving antiviral therapy (acyclovir 200mg five times a day) and an additional high zinc supplement. Patient education involved the pathogenesis of the disease, the importance of having adequate rest, taking nutritional food and reduction of stress. A week later, his conditioned was improved; the serology test revealed a reactive antiHSV-1 IgG (3.43) and the diagnosis was defined as RIH. As preventive strategies, he was agreed to take a long-term supplement of P.niruri extract 3 times daily regularly, take stress management and regular follow up of once a month. On the first follow up, he had an onset 10 days ago. Interval of recurrence episodes was 20 days with lower reactive antiHSV-1 Ig G (2.69). There were minute ulcer on labial mucosa, buccal mucosa and tongue. He was informed to continue P.niruri extract supplement and regular follow up. The patient was satisfied with the improvement compared with the condition in the past 3 months. Although, the disease still occurring, he confirmed there was a decrease in disease severity and prolonged onset interval. (Figure 3.)
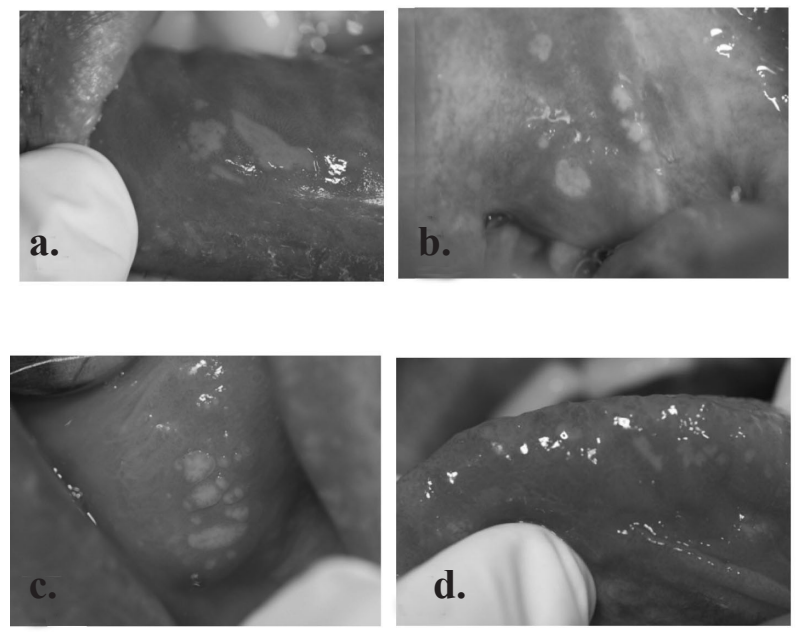

Figure 3. Multiple ulcers on (a)labial mucosa, (b)soft palate, (c)ventral and (d)lateral of the (Case 3) tongue.

\section{Discussion}

Patients presented in this paper had RIH for quite a long time and reoccurred within a short interval, with almost no healed period. Ulcers kept developing in different places before the previous ulcers had even healed. The serological tests confirmed that these patients were positive for RIH. Diagnosis can be made based on its clinical characteristic appearance but this might require experienced oral pathologist. Recurrent intraoral herpes is a self-limiting disease. Management for RIH is primarily symptomatic treatment, such as having adequate fluid intake, nutritious food, nutritional supplements, taking adequate rest and tainganalgesic/antipyretics and local antiseptics., 4, 6,8 Systemic medication in the form of antiviral therapy may be necessary for patients experiencing three to five episodes of HSV infection in a year or in combination with severe infection. ${ }^{6}$ Some clinicians suggest suppressive doses of antiviral therapy to decrease illness. ${ }^{1}$ As the management for our patients, symptomatic and antiviral therapy was given to each patient. Many research and literature suggested acyclovir is still efficacious and used at doses of 200 to $400 \mathrm{mg}$ taken 5 times daily., 9, 11,12 We used systemic (oral) acyclovir with suggested doses at 200mg taken 5 times daily.

The goal of management is to prevent the recurrences of disease. Onset of the disease depends on various factors thus it was important for each patient to recognize the triggers in order to control the recurrent disease. ${ }^{6} \mathrm{~A}$ 
thorough information of the disease to the patients is an important aspect of the management. ${ }^{2}$ The patients reported here confessed of having a physical and emotional stress on their life, we concluded that the trigger of all recurrences was stress. The trigger of stress was the most frequent trigger claimed by the patients as the main cause of the onsets. ${ }^{6}$ Stress, itself can cause an immune suppression that can lead to trigger RIH onset. These stressor results in the synthesis and release of a wide variety neuro-endocrine-derived peptides and hormones which can induce immune compromised. Reactivation of HSV increased in the patients who are immunologically compromised, suggesting a possible link between immune system and HSV latency. ${ }^{7}$ Michael and colleagues reported a study on mice and demonstrated that the function of resident CD8+ T-cells in the infected trigeminal ganglion was significantly compromised when mice were exposed to restraint stress. These CD8+ T-cells are capable to block the HSV reactivation in part through the production of antiviral cytokine interferon- $\gamma$ (IFN- $\gamma$ ). However, there might be another factors which can compromise T-cells or others cytokine. ${ }^{7}$ We educate patients to get enough bed rest, nutritious food and food supplement to restore physical health. Rich zinc supplementation also given to boost immune system. Zinc plays a role in increasing the number of T-cells, several studies have shown it prolonged the recurrent HSV episodes. ${ }^{6}$ Emotional stress or psychological stress was difficult trigger to overcome. Communication and empathy were needed to temporary ease the stress. We had suggested patients to look for help from the professionals but they refused and prefer to manage their own stress reduction by themselves.

Since stress reduction was more difficult to overcome, therefore another preventive strategy was through a long term oral medication in the form of antiviral therapy or supplementation therapy. ${ }^{6}$ Oral acyclovir proved to be an effective medication when taken as a prophylactic medication. It reduces the number and severity of the recurring disease. ${ }^{13}$ The protocols may include 200 to $400 \mathrm{mg}$ taken 5 times a day (1000-2000 mg daily) for as long as 6 months with regular follow up. ${ }^{9,10}$ Moreover, some of clinician suggested doses of $400 \mathrm{mg}$ acyclovir taken 2 times daily ( $800 \mathrm{mg}$ daily). ${ }^{3,13}$ We chose oral acyclovir $1000 \mathrm{mg}$ per day for the first patient, due to the severity of disease. She had more than 5 times episodes of recurrent ulcers for a year. Acyclovir is a synthetic acyclic analogue of 2'deoxyguanosine, activated by herpes enzyme and inhibit HSV DNA polymerase and HSV replication. ${ }^{12,13}$ On second and third cases, we chose Phyllanthusniruri"Meniran" (P.niruri) extract therapy with suggested dose 1 capsule, 3 times daily. We suggest the use of P.niruri supplementation was to reduce immune suppression caused by stresses. P.niruri extractwas commonly used as medicinal plants. ${ }^{14-16}$ P.niruriis a small, erect, annual herb that grows $30-40 \mathrm{~cm}$ in height and generally believe could improve specific and non-specific immune response and to be valuable in immunodeficiency conditions. ${ }^{16}$ Nworu et al. reported that extract of P.niruri is a potent murine lymphocytes mitogen, with significant increases in the expression of surface activation marker and proliferation of $\mathrm{B}$ and $\mathrm{T}$ lymphocytes including significant increases in production of IFN- $\gamma$. In vitro study showed immune activation potentials such as incrase cell proliferation on human peripheral blood mononuclear cells. ${ }^{14}$ Study on the P.niruri revealed that leaf samples of $P$. niruri contain manganese, cooper and zinc that are important for immune response regulation. ${ }^{15}$

On the first case, the preventive strategies had proven to be effective. There was a prolonged interval from continual recurrences from 3 to 5 days to 10 days interval. Unfortunately, she could not reduce her stress or attend regular follow up. The second and third case demonstrated a better progress. The patients had a better compliance on stress reduction. Intervals of recurrence on the second case was increasing from repeated recurrences to 14 days then to 17 days interval and revealed one recurrent episode during 3 months follow up. The third cases showed a prolonged interval from continuous onset in 3 months to 20 days interval. In addition, serological examination revealed a lower antiHSV-1 IgG on the last onset compare to the first onset. Unfortunately, regular follow up on clinical examination and regular serological test of antiHSV-1 could not be carried out due to financial problem.

Based on information gathered from all these patients, acyclovir and P.niruri extract showed a good improvement on reducing the number and severity of disease onsets. This report was in line with prolonged interval of recurrence, less amount of developing ulcer, shorten healing time also symptoms reduction although there were problems with patient compliance. It is believed that excellent compliance to take the medication regularly, willingness to meet psychological professionals and routine follow up, are the best strategies to prevent recurrence of RIH. The present case report showed the benefits of preventive strategies with stress counseling; long term acyclovir therapy and P.niruri extract supplementation on RIH patients with physiolocal and emotional stresses as the main trigger. 


\section{Conclusion}

Few strategies were described to prevent the disease onset such as stress reduction, long-term therapy of acyclovir and Phyllanthusniruri (Meniran) extract supplementation. Thus, result in decreasing the number and severity of disease onsets. Patient compliance is also needed to support the achievement of preventive strategies.

\section{Suggestion}

We suggest further clinical trial research to compare the effectiveness and capability of long-term acyclovir therapy in combination with P.niruri supplementation.

\section{References}

1. Siegel MA. Diagnostic and Management of Recurrent Herpes Simplex Infections. Journal of American Dental Association 2002;133:1245-9.

2. Woo SB, Greenberg MS. Ulcerative, Vesicular, and Bullous Lesions. In: Greenberg MS, Glick M, Ship JA, editors. Burket's Oral Medicine. 11 ed: BC Decker Inc; 2008. p. 41-6.

3. Drugge JM, Allen PJ. A nurse practitioner's guide to the management of herpes simplex virus- 1 in children. Pediatric Nursing. 2008;34(4):310-8.

4. Freeman ML, Sheridan BS, Bonneau RH, Hendricks RL. Psychological Stress Compromises CD8+ T Cell Control of Latent Herpes Simplex Virus Type 1 Infections. The Journal of Immunology. 2007;179:322-8.
5. Osterne RLV, Brito RGdM, Pacheco IA, Alves APNN, Sousa FB. Management of Erythema Multiforme Associated with Recurrent Herpes Infection: A case report. Journal of the Canadian Dental Association. 2009;75:597-601.

6. Pickett FA, Terezhalmy GT. Basic Principles of Pharmacology with Dental Hygiene Application. Philadelphia: Lippincott Williams and Wilkins; 2009.

7. Woo SB, Challacombe SJ. Management of Recurrent Oral Herpes Simplex Infection. Oral Surgery, Oral Medicine, Oral Pathology, Oral Radiology, Endodontology 2007;103 (supp11):S12-S8.

8. Haveles EB. Applied Pharmacology for The Dental Hygienist. Sixth ed. Virginia: Mostby Elsevier; 2011.

9. Amin ZA, Abdulla MA, Ali HM, Alshawsha MA, Qadirc SW. Assessment of In vitro antioxidant, antibacterial and immune activation potentials of aqueous and ethanol extracts of Phyllanthus niruri. J Sci Food Agric. 2012.

10. Gowrishankar R, Kumar M, Menon V, Divi SM, Saravanan M, Magudapathy P, et al. Trace Element Studies on Tinospora cordifolia (Menispermaceae), Ocimum sanctum (Lamiaceae), Moringa oleifera (Moringaceae), and Phyllanthus niruri (Euphorbiaceae) Using PIXE. Biol Trace Elem Res 2010;133:357-63.

11. Nworu CS, Akah PA, Okoye FBC, Proksch P, Esimone CO. The Effects of Phyllanthus niruri Aqueous Extract on the Activation of Murine Lymphocytes and Bone Marrow-Derived Macrophages. Immunological Investigations. 2010;39:245-67. 